\title{
Comportamento de risco para anorexia nervosa em universitários do curso de nutrição em uma instituição de ensino superior em Belém-PA
}

Risk behavior for nervous anorexia in university members of the nutrition course in a higher education institute in Belém-PA

\section{Comportamiento de riesgo para anorexia nerviosa en universitarios del curso de nutrición en una institución de enseñanza superior en Belém-PA}

Sheila Cristina Martins e Silva ${ }^{1 *}$, Diana Souza dos Santos ${ }^{1}$, Michele de Sousa Carneiro ${ }^{1}$, Ingryd Letícia Prazeres Carvalho ${ }^{1}$, Christian Nunes Aires ${ }^{1}$

\section{RESUMO}

Objetivo: Identificar os fatores de risco em adultos jovens do ensino superior para o desenvolvimento de anorexia nervosa em uma faculdade particular de Belém - PA. Metodologia: A população estudada foi constituída por 58 discentes matriculados no curso em graduação em nutrição do $3^{\circ}$ ao $8^{\circ}$ período. Para a obtenção dos dados foram utilizados avaliação antropométrica, questionários: sociodemográfico, EAT e escala de silhuetas corporais. Resultados: Foram achados percentuais elevados de risco para o desenvolvimento de Transtornos Alimentares (TA) (39,65\%), segundo alteração de percepção de imagem, insatisfação corporal e estado nutricional na população estudada, sendo que este risco foi maior entre os acadêmicos que apresentam eutrófia. O fato de a maioria dos universitários eutróficos apresentarem insatisfação de imagem por excesso de peso ou por desnutrição grau I é extremamente preocupante e indica que estes universitários, apesar de terem um IMC adequado, querem reduzir ainda mais suas silhuetas. Conclusão: Ao associar os resultados obtidos nos quatro instrumentos utilizados, a maioria dos avaliados se apresentou com risco para desenvolver TA como anorexia.

Palavras-chaves: Transtorno alimentar, Anorexia Nervosa, Adolescente.

\begin{abstract}
Objective: To identify risk factors in young adults in higher education for the development of anorexia nervosa in a private college in Belém - PA. Methodology: The studied population consisted of 58 students enrolled in the undergraduate course in nutrition from the 3rd to the 8th period. To obtain the data, anthropometric evaluation, questionnaires: sociodemographic, EAT and body silhouettes scale were used. Results: A high percentage of risk was found for the development of Eating Disorders (ED) (39.65\%), according to altered perception of image, body dissatisfaction and nutritional status in the studied population, and this risk was higher among the students who presented eutrophy. The fact that most of the eutrophic university students present image dissatisf action due to overweight or grade I mal nutrition is extremely worrying and indicates that these university students, despite having a suitable BMI, want to reduce their silhouettes even more. Conclusion: When associating the results obtained in the four instruments used, the majority of the evaluated ones presented with risk to develop ED like anorexia.
\end{abstract}

KEYWORDS: Eating Disorder, Anorexia Nervosa, Teenager.

${ }^{1}$ Faculdade Integrada Brasil Amazônia, FIBRA. *E-mail: nutricsheilamartins@gmail.com

SUBMETIDO EM: 9/2018 | ACEITO EM: 10/2018 | PUBLICADO EM: 12/2018 


\section{RESUMEN}

Objetivo: Identificar los factores de riesgo en adultos jóvenes de la enseñanza superior para el desarrollo de anorexia nerviosa en una facultad particular de Belém - PA. Metodología: La población estudiada fue constituida por 58 discentes matriculados en el curso en graduación en nutrición del 3 ol a $8^{\circ}$ período, en la institución de enseñanza superior. Para la obtención de los datos fueron utilizados evaluación antropométrica, cuestionarios: sóciodemográfico, EAT y escala de siluetas corporales. Resultados: Se encontraron porcentajes elevados de riesgo para el desarrollo de TA (39,65\%), según alteración de percepción de imagen, insatisfacción corporal y estado nutricional en la población estudiada, siendo que este riesgo fue mayor entre los académicos que presentan eutrofia. El hecho de que la mayoría de los universitarios eutróficos presentan insatisfacción de imagen por exceso de peso o por desnutrición grado I es extremadamente preocupante e indica que estos universitarios, a pesar de tener un IMC adecuado, quieren reducir aún más sus siluetas. Conclusión: Al asociar los resultados obtenidos en los cuatro instrumentos utilizados, la mayoría de los evaluados se presentó con riesgo para desarrollar trastornos alimentarios como anorexia.

PALABRAS CLAVES: Trastorno alimentício, Anorexia nerviosa, Adolescente.

\section{INTRODUÇÃO}

A alimentação é caracterizada por ser um fenômeno de grande complexidade que envolve vários aspectos: psicológicos, biológicos, fisiológicos, sociais, econômicos, científicos, políticos e culturais fundamentais na dinâmica da evolução das sociedades. No ato de alimentar-se está inserido a história de cada indivíduo, suas lembranças, sentimentos, conflitos, estado emocional e social, e sua relação de forma ampla com o alimento (CANDIDO, et al., 2010; PROENÇA, 2010; SILVA, 2013).

Apesar da alimentação acarreta inúmeros benefícios a saúde, a relação que o indivíduo estabelece com o alimento está relacionado com o desenvolvimento de doenças, no qual vem sendo associada com transtorno alimentar (TA). O desenvolvimento de TA está relacionado com dietas extremamente restritas, consumo alimentar inadequado, insatisfação corporal, comportamento compulsivo, ansiedade e medo de engordar, compensação de problemas emocionais com a comida e radicalismo com as escolhas dos alimentos $e$ calorias (CARAM e LAZARINE, 2013; NUNES et al., 2017).

Os TA são desordens psiquiátricas que ocorrem, na maioria das vezes em adolescentes e adultos jovens do sexo feminino, cerca de $90 \%$ dos af etados, e podem levar a grandes prejuízos biológicos e psicológicos e ao aumento de morbidade e mortalidade. Eles são de origem multifatorial como anteriormente citados. Estes aspectos determinam as dimensões necessárias para o diagnóstico e o tratamento, devido à complexidade da condição clínica, exige-se uma abordagem integral e multiprofissional, ou seja, uma equipe formada por médico, psicólogo, nutricionista, psiquiatra, enf ermeiro, dentre outros (SILVA e MURA, 2014).

Desta forma, a presente pesquisa tem como objetivo identificar os fatores de risco para o desenvolvimento de transtorno alimentar em universitários do curso de nutrição, de acordo com o transtorno alimentar conhecido como anorexia nervosa.

\section{METODOLOGIA}

Trata-se de um estudo do tipo descritivo transversal, com adultos jovens dentro de uma faixa etária de 18 a 23 anos de idade do $3^{\circ}$ ao $8^{\circ}$ semestre do curso de nutrição, matriculados regularmente em uma faculdade particular, Belém - PA. Para a elaboração do referencial teórico foi realizada pesquisa em livros, artigos científicos na base de dados da Scielo (Scientific Electronic Library), Biblioteca Virtual de Saúde, ABESO (Associação Brasileira para Estudo da Obesidade e Síndrome Metabólica), WHO (World Health Organization), IBGE (Instituto Brasileiro de Geografia e Estatística) e DeCS (Descritores em Ciências da Saúde). 
Foi adotado um método quantitativo em função de verificar as variáveis pré-determinadas, buscando verificar sua existência, relação ou influência sobre outra variável (TERENCE e FILHO (2006).

\section{Seleção da Amostra}

A pesquisa foi realizada na Faculdade Integrada Brasil Amazônia (FIBRA), no qual foi desenvolvida com adultos jovens de ambos os sexos, na faixa etária entre 18 e 23 anos, matriculados do 3 a ao 8 o semestre de 2018, período matutino, no mês de abril, acadêmicos do curso de nutrição.

O número total de alunos matriculados nesta Instituição de Ensino Superior (IES) era de 201 indivíduos. Desta forma, aceitando-se uma margem de erro de $5 \%$, com um nível de confiança de até $95 \%$, a amostra para esta pesquisa foi de 58 indivíduos, número este baseado na fórmula de cálculo de (SANTOS, 2016).

Fórmula de cálculo:

$$
n=\frac{N \cdot Z^{2} \cdot p \cdot(1-p)}{Z^{2} \cdot p \cdot(1-p)+e^{2} \cdot(N-1)}
$$

Onde:

$\mathrm{n}$ - amostra calculada

$\mathrm{N}$ - população

Z - variável normal padronizada associada ao nível de confiança

$p$ - verdadeira probabilidade do evento

e - erro amostral

Inicialmente, para a seleção da amostra, foi utilizada uma lista fornecida pela secretaria acadêmica, onde o número de alunos matriculados, até aquela data, era de 201, dispostos por período, nome do aluno, sexo e data de nascimento, onde foi possível estabelecer os alunos que participaram da pesquisa de acordo com a idade preconizada (entre 18 e 23 anos), onde então, foi elaborada uma nova listagem somente com os alunos previamente selecionados, para facilitar a identificação deles onde foi dado prosseguimento à entrega de um Termo de Consentimento e Livre Esclarecimento (TCLE), posteriormente, ao recebimento deste termo devidamente assinado pelos pesquisados, para então ser dado prosseguimento à coleta de dados.

Foram inseridos os dados através dos questionários e codificados para serem inclusos em tabela Excel, e realizado a estatística descritiva.

\section{Aspectos Éticos}

O projeto foi encaminhado ao CEP, com o objetivo de cumprir o disposto na Resolução nำ466/12 do Conselho Nacional de Saúde, aprovado, e posteriormente se deu continuidade ao mesmo. Onde foi apresentado o TA para cada participante da pesquisa. A pesquisa foi realizada de acordo com a resolução no 466/12, do conselho nacional de saúde, após aprovação pelo comitê de ética em pesquisa da faculdade integrada brasil Amazônia em BELEM-PA, sob o parecer ํo 2.582.440.

\section{RESULTADOS}

Foram analisados 58 universitários onde prevaleceu o sexo feminino com $84 \%$, a faixa etária da pesquisa que predominou $72 \%$ de 21 a 23 anos, e o período que predominou foi o 3 o período $36 \%$ de acordo com a Tabela 1, onde utilizou-se o questionário sóciodemográfico. 
Tabela 1 - Frequencia absoluta e relativa dos gêneros, faixa etária e períodos dos alunos de nutrição de uma faculdade particular Belém/Pará.

\begin{tabular}{lcc}
\hline Gênero & $\mathbf{N}$ & $\%$ \\
\hline Masculino & 9 & $16 \%$ \\
Feminino & 49 & $84 \%$ \\
Total & 58 & $100 \%$ \\
\hline Faixa etária & $\mathbf{N}$ & $\%$ \\
$18-20$ & 16 & $28 \%$ \\
$21-23$ & 42 & $72 \%$ \\
Total & 58 & $100 \%$ \\
\hline Período dos universitários & $\mathbf{N}$ & $\%$ \\
\hline 3o período & 21 & $36 \%$ \\
5o período & 5 & $9 \%$ \\
70 período & 16 & $28 \%$ \\
8o período & 16 & 280 \\
Total & 58 & $100 \%$ \\
\hline
\end{tabular}

Fonte: Protocolo de pesquisa, 2018.

Constatou-se sobre o questionamento sócio demográfico com a seguinte pergunta: "você pratica exercício físico", nessa variável obteve-se a maioria $52 \%(n=30)$; dos acadêmicos que não praticam exercício físico,e obteve-se segundo a variável " tenho medo de engordar ou ficar mais gordo", obteve-se a predominância de $71 \%(n=41)$, que disseram ter medo de engordar, e conforme a variável " percepção com o físico te leva a fazer dieta”, obteve-se a predominância de 60\% ( $n=35)$, disseram que sim, tem preocupação em fazer dieta, de acordo com a Tabela 2.

Tabela 2 Frequência absoluta das repostas do questionário sócio demográfico.

\begin{tabular}{lcc}
\hline "Você pratica exercício fisico" & $\mathbf{N}$ & $\%$ \\
\hline Sim & 28 & $48 \%$ \\
Não & 30 & $52 \%$ \\
Total & 58 & $100 \%$ \\
\hline "Você tem medo de engordar" & $\mathbf{N}$ & $\%$ \\
\hline Sim & 41 & $71 \%$ \\
Não & 17 & $29 \%$ \\
Total & 58 & $100 \%$ \\
\hline "Você se preocupa em fazer dieta" & $\mathbf{N}$ & $\%$ \\
\hline Sim & 35 & $60 \%$ \\
Não & 23 & $40 \%$ \\
\hline Total & 58 & $100 \%$ \\
\hline "Você se sente insastifeito com seu corpo" & $\mathbf{N}$ & $\%$ \\
Sim & 31 & $53 \%$ \\
Não & 27 & $47 \%$ \\
\hline Total & 58 & $100 \%$ \\
\hline
\end{tabular}

Fonte: Protocolo pesquisa, 2018.

De acordo com o questionário sociodemográfico verificou-se segundo a variável "atualmente como você se sente", predominou 44\% ( $n=18)$, acadêmicos abaixo do peso, de acordo com o (Gráfico 1). 
Gráfico 1. Atualmente como você se sente.

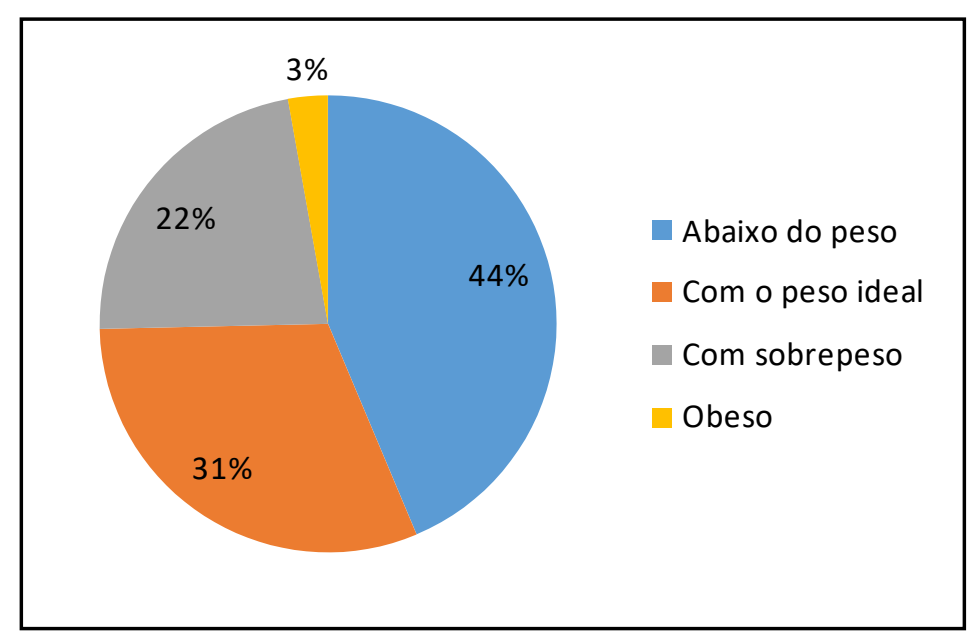

Fonte: protocolo pesquisa, 2018.

A antropometria foi utilizada para identificar o estado nutricional dos universitários, servindo como um bom diagnostico através do IMC, portanto segundo a variável de avaliação nutricional, verificou-se a predominância de $72 \%(\mathrm{~N}=42)$ de eutrófia. E segundo a avaliação de percepção da imagem corporal predominou com $28 \%$ $(\mathrm{N}=16)$ com sobrepeso, de acordo com o gráfico 2.

Gráfico 2 - Percepção e relação da imagem corporal e IMC.

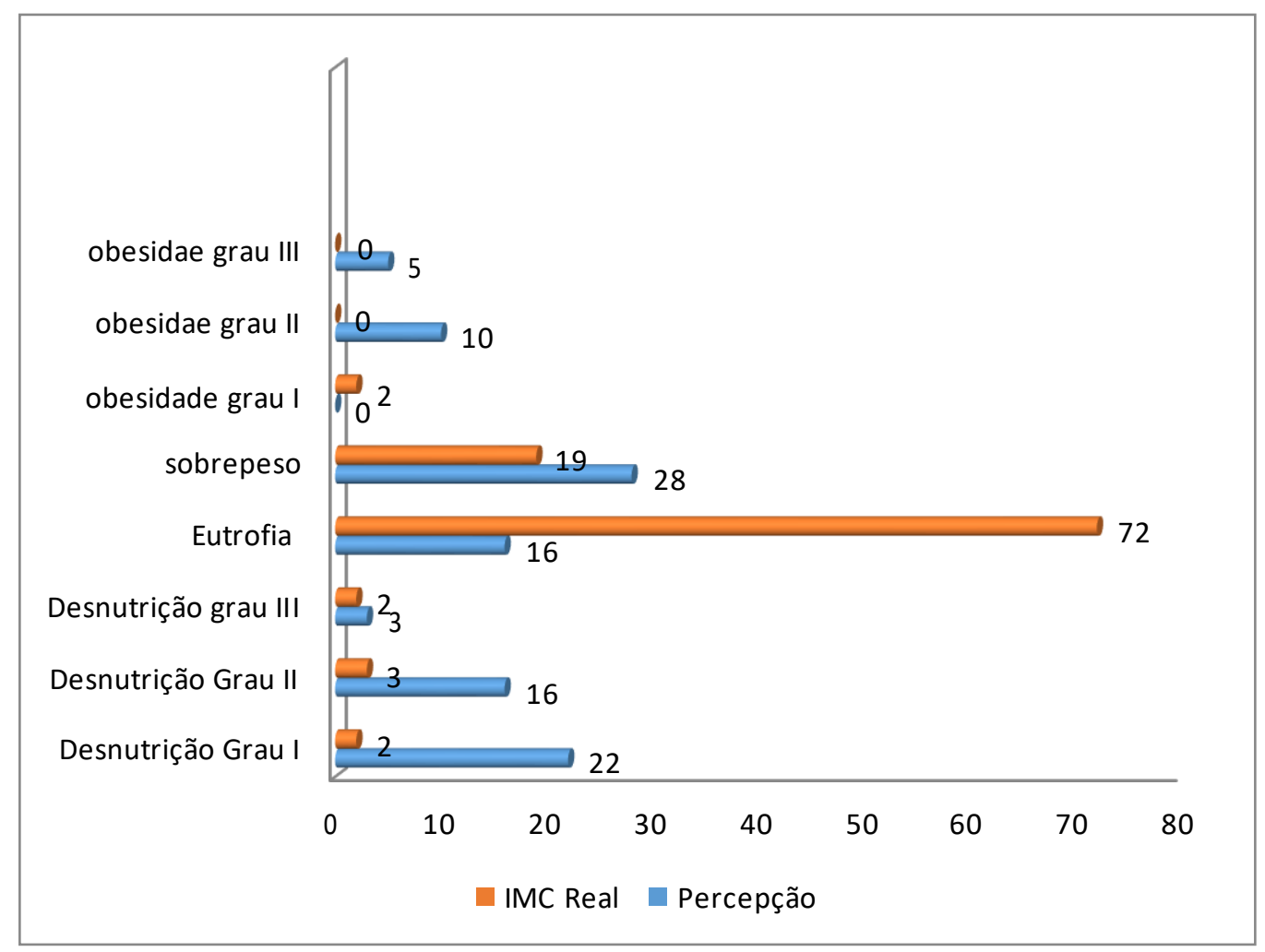

Fonte: Protocolo da pesquisa, 2018. 
Segundo os dados do EAT (teste de atitudes alimentares), o resultado da amostra foi $39,65 \%$ ( $n=23$ ), portanto, EAT positivo. Dentre os 58 questionários, obteve-se 23 acadêmicos com resultado maior que 20, que é o ponto mínimo para ser considerado como fator de risco de anorexia. Fato que justifica os resultados do estado nutricional de acordo com IMC, que se obteve $72 \%(n=42)$ com eutrofia; e o resultado das silhuetas com apenas $16 \%(n=9)$ que se veem com eutrofia.

\section{DISCUSSÃo}

Os transtornos alimentares são distúrbios psiquiátricos relacionados ao comportamento alimentar com consequências nutricionais muito graves e de forte impacto na saúde dos indivíduos. Os resultados obtidos nesta pesquisa são importantes indicadores de fatores predisponentes de anorexia nervosa na população estudada.

Foram avaliados 58 universitários, do período do $3^{\circ}, 5^{\circ}, 7^{\circ}$ e $8^{\circ}$ semestre, sendo assim a maioria possuiem conhecimento para identificar fatores de risco para TA, Anoreia nervosa - NA, semelhante ao encontrado no estudo de Kirsten et al. (2009) e Reis e Soares (2017), que obteve como resultado que os indivíduos que tem alguma preocupação com o controle do peso e com a imagem corporal, optam pelo curso de nutrição e talvez este seja um dentre vários motivos, que justifiquem os elevados percentuais de risco para o desenvolvimento de TA.

Em relação à classificação do IMC a maioria se encontra com estado nutricional eutrofico, obtiveram resultados de sobrepeso e magreza grau I, II e III, estes achados foram parecidos com os resultados de Minzon (2010), Souza et al. (2010), onde 75\% dos estudados apresentam eutrófia e 16,9\% sobrepeso, 0,7\% com magreza I, II, III. É importante ressaltar que o Índice de Massa Corporal (IMC) não reflete o estado nutricional do adulto de forma isolada, visto que pode subestimar a massa gorda e a massa magra.

Quando questionados "se pratica exercício físico", a maioria dos participantes não praticam exercício físico e são mais insatisf eitos com a imagem comoral comparados com os ditos sedentários, corroborando com o estudo de Laus et al. (2006), desta forma, ressalta-se a importância da prática de exercício físico para melhorar ou manter a qualidade de vida em todas as faixas etárias de idade, como enfatiza os estudos de Silva, et al., (2010).

De acordo com o elevado percentual de eutrofia $72 \%$, o mesmo não reflete os dados de insatisfação com a imagem corporal, que obteve $16 \%$ apenas se vêm com eutrofia, nem o Teste de Atitude Alimentar - EAT positivo 39,65\%, o que pode ser confirmado através das pesquisas realizadas por Batista e Bailão (2016), Reis e Soares (2017), onde $71,73 \%$ dos pesquisados foram classificados como eutróficos pelo IMC e $38,8 \%$ EAT.

Em relação "a fazer dieta" $60 \%$, dos pesquisados faziam dieta o que pode se relacionar com a percepção da imagem que resultou em $22 \%$ de desnutrição. Outros estudos com universitários apontam para a insatisf ação corporal avaliada através da escala de silhueta variando de $61,2 \%$ a $78,8 \%$, na presente pesquisa o resultado foi de $94 \%$ o que corrobora com os estudos de Reis e Soares (2017).

Dos acadêmicos pesquisados sobre "o medo de engordar", a maoria relatou ter medo de engordar e apresentavam estado nutricional eutrofico, porém quando foram questionados "de como se sentia atualamente", descreveram estar abaixo do peso, contrapondo-se ao resultado de eutrofia na avaliação antropométrica, como constatado nos estudos de Minzon (2010) sobre este questionamento.

A amostra de que foi objeto desta pesquisa descreveu uma realidade concordante com o presumido inicialmente sobre os fatores de risco para TA - AN. A prevalência de indicativo de TA foi de 39,65\%, e apesar da maioria da amostra estar com IMC eutrofico, houve um percentual alto (53\%) de insatisfação corporal, valores similares ao identificado no estudo Reis e Soares (2017), que foi possível observar um perfil preocupante entre os acadêmicos do curso de nutrição IMC 72,7\%, EAT 23,7\% a 31,1\% e insatisfação corporal $69,7 \%$. 


\section{CONCLUSÃO}

Ao associar os resultados obtidos nos quatro instrumentos utilizados, 23 universitários apresentaram risco para desenvolver TA como anorexia nervosa, sendo que esta associação pode ser interessante na triagem de indivíduos suscetíveis ao desenvolvimento destes transtornos. Os resultados deste estudo reafirmam a necessidade da realização de medidas de conscientização e prevenção de TA com estudantes de Nutrição, bem como a realização de novos estudos que investiguem mais profundamente o tema em questão. A alimentação tem um papel importante no desenvolvimento e manutenção de TA, trata-se de doenças complexas e que precisa de um tratamento multiprofissional ef etivo, e o nutricionista é o único profissional qualificado a executar estratégias nutricionais visando à recuperação do estado nutricional desses pacientes, orientando e aconselhando sobre uma dieta adequada. O acompanhamento nutricional tem como objetivo desmistificar hábitos alimentares inadequados e constituir uma relação adequada com o alimento. Sendo assim o nutricionista está inserido em qualquer esfera de apoio a esses pacientes, desde a prevenção até o tratamento.

\section{REFERÊNCIAS}

1. BATISTA EA, BAILÃO MS. Avaliação do risco de desenvolvimento de transtornos alimentares em adolescentes da cidade de Bebedouro SP. Revista Fafibe on-line, 2016;20 (1);166-177.

2. BRASIL. Lei n. 8.069, de 13 de julho de 1990. Dispõe sobre o Estatuto da Criança e do Adolescente e dá outras providências. Lex:Estatuto da Criança e do Adolescente. Brasília: senado Federal, 1990.

3. CANDIDO CC, GOMES CET, SANTOS EC, GAMES GMO et al. Nutrição: guia prático. Transtornos alimentares na adolescência. 3 ed. São Paulo: látria, 2010. p. 49-50, 2010.

4. CARAM ALA, LAZARINE IF. Atitudes alimentares em universitários dos cursos de Nutrição, Educação Física e Psicologia de uma instituição privada. Rev. Inst. Cienc. Saúde, 2013;30(1):71-73.

5. KIRSTEN VR, FRATTON F, PORTA NBD. Transtornos alimentares em alunas de nutrição do Rio Grande do Sul. Rev. Nutr, 2009;22(2): 22-27.

6. LAUS MF, ZANCUL MS, MARTINS TM, KAKESHITA IS, ALMEIDA SS. Percepção da imagem corporal e estado nutricional em estudantes de nutrição. Alimentos e Nutrição, 2006;17(1): 85-86.

7. MINZON JC. Fatores de risco para o desenvolvimento de transtornos alimentares entre adolescentes de uma escola pública de Campo Grande - MS. Dissertação (Mestrado em psicologia) - Universidade Católica Dom Bosco do Mato Grosso do Sul, Campo Grande, 2010:92 p.

8. NUNES LG, SANTOS MC, SOUZA AA. Fatores de risco associados ao desenvolvimento de bulimia nervosa em estudantes universitários: uma revisão integrativa. HU Revista, 2017; 43(1):61-69.

9. PIZZETTA ZO, ARBOSA A, SANT'ANA PT, MARCIANO GCS et al. Associação da imagem corporale transtornos alimentares em adolescentes de Minas Gerais (Brasil). Nutr. Clín. Diet. Hosp, 2015:35(2):48-54.

10. PROENÇA RPC. Alimentação e globalização: algumas reflexões. Cienc. Cult, 2010;62(4): 43-46.

11. REIS S, SOARES,LP. Estudantes de nutrição apresentam risco para transtorno alimentar. Revista Brasileira de Ciência da Saúde, 2017;21(4): 281-290.

12. SANTOS GEO. Fórmula de cálculo: Cálculo amostral: calculadora on-line, 2016.

13. SILVA, J. A. T. . Nutrição nos transtomos psiquiáticos. 1. ed. viçosa:A.S.SISTEMAS, 2013. v. 1.250p.

14. SILVA RS., SILVA I, SILVA RA, SOUZA LTE. Atividade física e qualidade de vida. Ciência \& Saúde Coletiva, 2010;15(1): 115-120.

15. SILVA SMCS, MURA JDP. Tratado de alimentação, nutrição e deitoterapia. In.: Terapia nutricional em anorexia e bulimia nervosa. $2^{\mathrm{a}}$ ed. São Paulo: Roca, 2014. Cap. 44, V. 2, p. $759-762,2014$.

16. TERENCE ACF, FILHO EE. Abordagem quantitativa, qualitativa e a utilização da pesquisa-ação nos estudos organizacionais. XXVI ENERGEP - Fortaleza, CE, Brasil, 2006;26(1): 1 - 4. 